\title{
Properties of Suprathermal-through-energetic He Ions Associated with Stream Interaction Regions Observed over the Parker Solar Probe's First Two Orbits
}

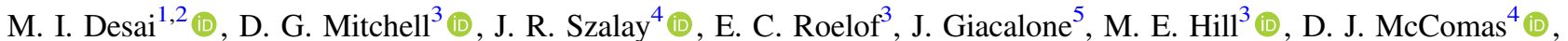
E. R. Christian ${ }^{6}$ (1) , N. A. Schwadron ${ }^{7}$ (1), R. L. McNutt Jr. ${ }^{3}$, M. E. Wiedenbeck ${ }^{8}$, C. Joyce ${ }^{4}$ (10), C. M. S. Cohen ${ }^{8}$, R. W. Ebert ${ }^{1,2}$ (1),

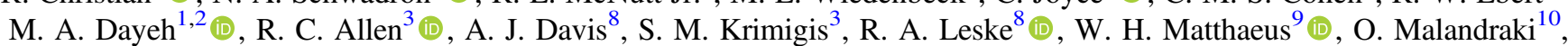
R. A. Mewaldt ${ }^{8}$, A. Labrador $^{8}$, E. C. Stone ${ }^{8}$, S. D. Bale ${ }^{11}$ (1), M. Pulupa ${ }^{11}$ (D), R. J. MacDowall ${ }^{6}$ (i), and J. C. Kasper ${ }^{12}$ (D)

${ }^{1}$ Southwest Research Institute, 6220 Culebra Road, San Antonio, TX 78238, USA

2 Department of Physics and Astronomy, University of Texas at San Antonio, San Antonio, TX 78249, USA

3 Johns Hopkins University/Applied Physics Laboratory, Laurel, MD 20723, USA

${ }^{4}$ Department of Astrophysical Sciences, Princeton University, NJ 08544, USA

5 The University of Arizona, Lunar and Planetary Laboratory, Tucson, AZ 85721, USA

${ }^{6}$ NASA Goddard Space Flight Center, Greenbelt, MD 20771, USA

${ }^{7}$ University of New Hampshire, 8 College Road, Durham, NH 03824, USA

${ }^{8}$ California Institute of Technology, Pasadena, CA 91125, USA

${ }^{9}$ University of Delaware, Newark, DE 19716, USA

${ }^{10}$ Institute for Astronomy, Astrophysics, Space Applications \& Remote Sensing (IAASARA), National Observatory of Athens, Greece

${ }^{11}$ University of California, Berkeley, Space Sciences Laboratory, Berkeley, CA 94720, USA

${ }^{12}$ University of Michigan, Ann Arbor, MI 48109, USA

Received 2019 December 6; revised 2019 December 27; accepted 2019 December 27; published 2020 February 3

\begin{abstract}
The Integrated Science Investigation of the Sun (IS $\odot$ IS) suite on board NASA's Parker Solar Probe (PSP) observed six distinct enhancements in the intensities of suprathermal-through-energetic $\left(\sim 0.03-3 \mathrm{MeV}^{\text {nucleon }}{ }^{-1}\right)$ He ions associated with corotating or stream interaction regions (CIR or SIR) during its first two orbits. Our results from a survey of the time histories of the He intensities, spectral slopes, and anisotropies and the event-averaged energy spectra during these events show the following: (1) In the two strongest enhancements, seen at 0.35 and $0.85 \mathrm{au}$, the higher-energy ions arrive and maximize later than those at lower energies. In the event seen at $0.35 \mathrm{au}$, the He ions arrive when PSP was away from the SIR trailing edge and entered the rarefaction region in the highspeed stream. (2) The He intensities either are isotropic or show sunward anisotropies in the spacecraft frame. (3) In all events, the energy spectra between $\sim 0.2$ and $1 \mathrm{MeV}$ nucleon ${ }^{-1}$ are power laws of the form $\propto E^{-2}$. In the two strongest events, the energy spectra are well represented by flat power laws between $\sim 0.03$ and $0.4 \mathrm{MeV}$ nucleon $^{-1}$ modulated by exponential rollovers between $\sim 0.4$ and $3 \mathrm{MeV}$ nucleon ${ }^{-1}$. We conclude that the SIRassociated He ions originate from sources or shocks beyond PSP's location rather than from acceleration processes occurring at nearby portions of local compression regions. Our results also suggest that rarefaction regions that typically follow the SIRs facilitate easier particle transport throughout the inner heliosphere such that low-energy ions do not undergo significant energy loss due to adiabatic deceleration, contrary to predictions of existing models.
\end{abstract}

Unified Astronomy Thesaurus concepts: Solar wind (1534); Corotating streams (314); Interplanetary particle acceleration (826)

\section{Introduction}

Energetic particle intensity enhancements associated with recurrent high-speed streams or corotating interaction regions (CIRs) have been routinely observed at interplanetary spacecraft near Earth orbit during periods of low solar activity since the 1960s (e.g., Bryant et al. 1963; Richardson et al. 1993). Stream interaction regions (SIRs) are typically formed when fast solar wind streams emanating from lower-latitude excursions of coronal holes overtake the slower solar wind originating from coronal streamers; SIRs become CIRs once they have persisted for at least one solar rotation (e.g., Gosling et al. 1981). Measurements obtained by Pioneer 10 and Pioneer 11 near the ecliptic plane (Barnes \& Simpson 1976; McDonald et al. 1976; Smith \& Wolfe 1976) and later by Ulysses at higher heliographic

Original content from this work may be used under the terms of the Creative Commons Attribution 4.0 licence. Any further distribution of this work must maintain attribution to the author(s) and the title of the work, journal citation and DOI. latitudes (e.g., Gosling et al. 1993; Simnett et al. 1994; Desai et al. 1997, 1999) confirmed that such CIRs are typically bounded by forward and reverse waves that steepen into shocks with increasing heliocentric distance from the Sun (see review by Richardson 2018). Until the late 1990s, it was believed that most of the recurrent energetic particles observed at 1 au are accelerated at distant corotating shocks and that some fraction of the accelerated particles undergo adiabatic deceleration as they propagate into the inner heliosphere before being detected at near-Earth satellites (e.g., Gloeckler et al. 1979; Fisk \& Lee 1980). These earlier models predicted that, depending on the shock distance from $1 \mathrm{au}$, ions below $\sim 0.5 \mathrm{MeV}$ nucleon $^{-1}$ would not be observed near Earth orbit and the differential energy spectrum would exhibit a turnover at lower energies because these ions suffer significant energy losses due to adiabatic deceleration as they propagate back toward Earth against the outflowing solar wind (Fisk \& Lee 1980).

Advanced instrumentation on Wind and Advanced Composition Explorer (ACE), both launched in the 1990s, showed that 
the energy spectra in CIR-associated particle events continued as power laws down to $\sim 0.03 \mathrm{MeV}^{\text {nucleon }}{ }^{-1}$ (e.g., Mason et al. 1997, 2008, 2012), indicating either that the earlier models overestimated the amount of energy losses due to adiabatic deceleration or that the particles seen at 1 au originated from sources closer to the spacecraft and are accelerated locally either via stochastic mechanisms (e.g., Schwadron et al. 1996; Chotoo et al. 2000) or via processes acting at compression regions without shocks (Chotoo et al. 2000; Giacalone et al. 2002). Indeed, Ebert et al. (2012a) showed that the peak $\sim 0.1-0.8 \mathrm{MeV}$ nucleon $^{-1} \mathrm{He}$ intensities in 73 CIR-associated events at ACE and STEREO were well correlated with magnetic compression ratios regardless of whether the reverse shocks were observed locally. Furthermore, Ebert et al. (2012b) found that the flow direction of $\sim 0.06-0.95 \mathrm{MeV}$ nucleon ${ }^{-1} \mathrm{He}$ in the solar wind plasma frame reverses from antisunward to sunward at the trailing edges of CIRs that were bounded by reverse shocks or associated with strong compression regions. Such flow reversals were not observed in events associated with weaker compression regions - here the flow remained predominantly sunward. In summary, the 1 au studies provided compelling evidence that suprathermal He ions below $\sim 1 \mathrm{MeV}$ nucleon $^{-1}$ can occasionally be accelerated locally near the trailing edges of well-formed compression regions.

Since only a handful of CIR-associated particle enhancements were observed by Helios inside 0.6 au (Van Hollebeke et al. 1978), the majority of observations of these types of particle events have been restricted to spacecraft located at or beyond Earth orbit. Furthermore, Helios did not have particle instruments capable of distinguishing between $\mathrm{He}$ and protons at energies below $\sim 0.4 \mathrm{MeV}^{\text {nucleon }}{ }^{-1}$. The Integrated Science Investigation of the Sun (IS $\odot$ IS; see McComas et al. 2016) suite on Parker Solar Probe (PSP; Fox et al. 2016) carries appropriate instrumentation (Section 2) and thus provides the first-ever measurements of suprathermal-through-energetic $\mathrm{He}$ ions with energy between $\sim 0.03$ and $2 \mathrm{MeV}$ nucleon ${ }^{-1}$ during one intensity enhancement that was associated with a CIR or SIR in the inner heliosphere at $\sim 0.35 \mathrm{au}$. We compare and contrast the time histories of the intensities, particle flow directions as measured from the anisotropies, and spectral slopes, and we analyze the shapes of the event-averaged differential energy spectra during this event with measurements during the other five SIR-associated intensity enhancements observed by PSP beyond $\sim 0.8 \mathrm{au}$. We highlight our new findings and compare with expectations from contemporary CIR-associated particle acceleration and transport models and theories.

\section{Instrumentation and Data Analysis}

Following a successful launch on 2018 August 12, PSP is now in an orbit around our Sun that will allow unprecedented observations of the inner regions of its outer atmosphere (Fox et al. 2016). Over the next $5 \mathrm{yr}$, PSP will use six more Venus flybys to slowly decrease its perihelion to within $\sim 9$ solar radii $\left(R_{\mathrm{s}}\right)$. PSP has now completed its first three orbits and, with a perihelion of $\sim 35 R_{\mathrm{s}}$, has already set the record for being the closest man-made object ever to have explored the inner heliosphere. PSP has four instrument suites that provide detailed and comprehensive measurements of the in situ solar wind, the embedded electromagnetic fields, and energetic particles, as well contextual white-light images of the solar corona and the inner heliosphere: the Solar Wind Electrons,
Alphas, and Protons Investigation (SWEAP, Kasper et al. 2016); the Electromagnetic Fields Investigation (FIELDS; Bale et al. 2016); the IS $\odot$ IS (McComas et al. 2016); and the Wide field Imager for Solar Probe (Vourlidas et al. 2016).

The IS $\odot$ IS suite comprises two instruments, namely, the lowenergy Energetic Particle Instrument (EPI-Lo; Hill et al. 2017) and the high-energy Energetic Particle Instrument (EPI-Hi), which collectively provide comprehensive measurements of the energy spectra, arrival directions, and composition of $\mathrm{H}-\mathrm{Fe}$ ions from $\sim 0.02$ up to $200 \mathrm{MeV}$ nucleon $^{-1}$, as well as of the energy spectra and arrival directions of $0.025-6 \mathrm{MeV}$ electrons. Where available, we also use data from the PSP/FIELDS (Bale et al. 2016) and PSP/SWEAP (Kasper et al. 2016) instrument suites.

In this paper, we use $\sim 0.03$ to $3 \mathrm{MeV}$ nucleon ${ }^{-1} \mathrm{He}$ data from IS $\odot$ IS, and we refer the reader to McComas et al. (2016) for more details about the EPI-Hi and EPI-Lo instruments. EPI-Hi comprises one single-ended and two double-ended telescopes with a stack of silicon detectors that enable species identification via the standard $d E / d x$ versus residual energy technique. The two high-energy telescopes, HETA and HETB, measure ions above $\sim 10 \mathrm{MeV}$ nucleon $^{-1}$, while ions with energies below $\sim 20 \mathrm{MeV}$ nucleon $^{-1}$ are measured by low-energy telescopes, LET1 (a double-ended sensor) and LET2 (a single-ended sensor). EPI-Lo has eight wedges with 10 apertures each that together cover a hemisphere of the sky. Ions above $\sim 0.02 \mathrm{MeV}$ nucleon $^{-1}$ entering each aperture generate secondary electrons as they pass through the start foils. The ions then continue and strike a solidstate detector (SSD) where they generate secondary electrons for the stop signal. The SSD also measures the residual kinetic energy of the ion. The coincidence between start, stop, and energy signals provides triple coincidence measurements, and the measured time of flight (TOF) and residual energy $(E)$ are used to calculate the ion mass. In this paper we only use the triple coincidence $\mathrm{He}$ data, which are binned in predefined incident energy ranges and species identities using onboard lookup tables based on preflight calibration measurements. These lookup tables are likely to change over the duration of the mission, and especially after the species tracks in TOF versus $E$ matrix get populated when PSP observes more intense SEP events (see McComas et al. 2016).

The triple coincidence data have the inherent advantage of having the lowest background among all of the EPI-Lo data products (e.g., TOF only or SSD only; see Giacalone et al. 2020; Hill et al. 2020) but are disadvantaged because of poor counting statistics. To accumulate the minimum counting statistics (i.e., at least 1 count) during the time interval, look direction, or energy range of interest, we average the $\mathrm{He}$ intensities over multiple aperture, as well as over broader energy ranges and longer time intervals. For time averaging, we average either over 2 or $4 \mathrm{hr}$ time intervals, and we sum the $\mathrm{He}$ counts detected between a factor of 2 in energy range, e.g., $0.031-0.062 \mathrm{MeV}$ nucleon $^{-1}$, and so on. In order to analyze the arrival directions of the He ions during the events of interest, we obtain the unit vector along the look direction of each aperture in a $P S P$-centered RTN coordinate system, where $+R$ is along the radial direction to the Sun, $+T$ is the direction of the component of the spacecraft velocity normal to $R$, and $+N$ completes the right-handed triad. For each event, we then obtain average $\mathrm{He}$ intensities in three directions, namely, (1) sunward, defined here as all apertures with look directions within $60^{\circ}$ of the $+R$ direction; (2) transverse, defined here as all apertures with look directions between $60^{\circ}$ and $120^{\circ}$ of the 

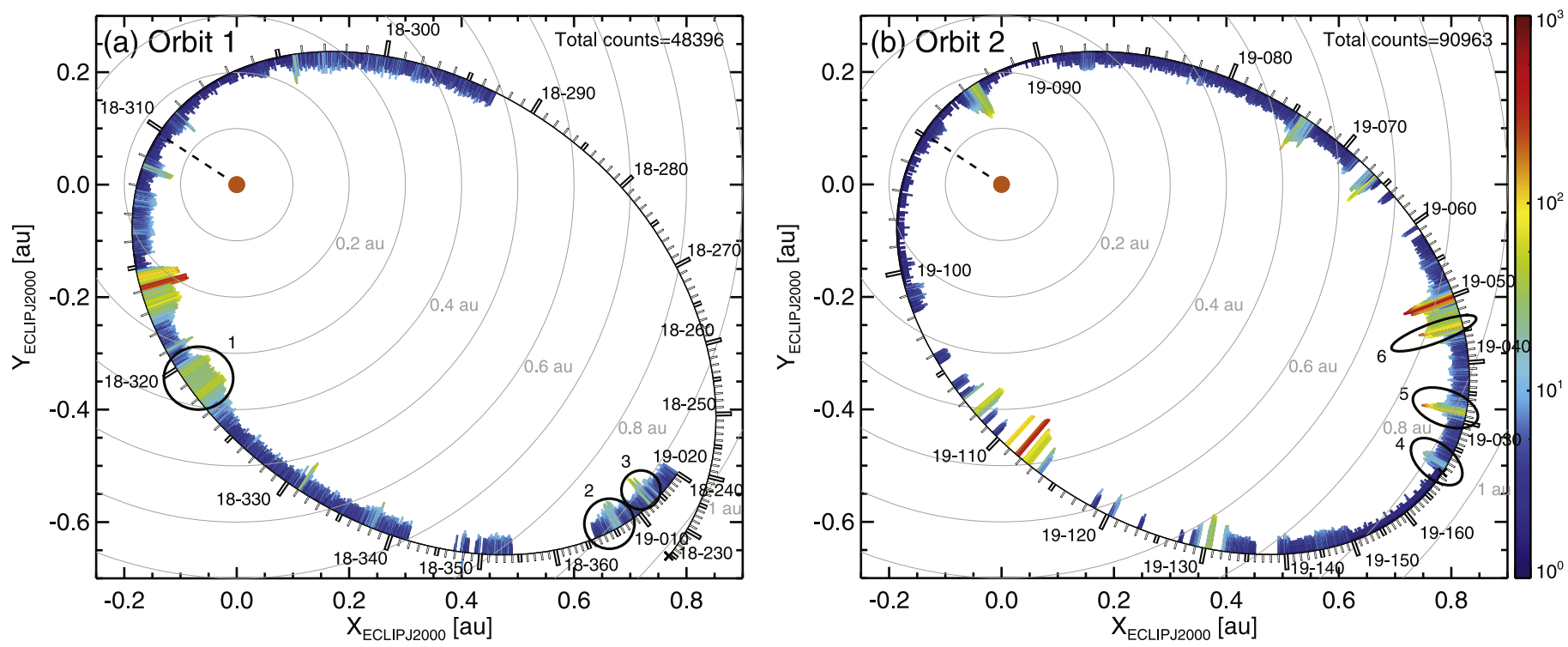

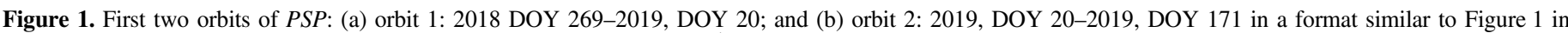

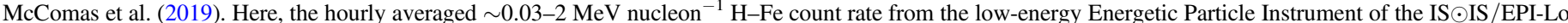

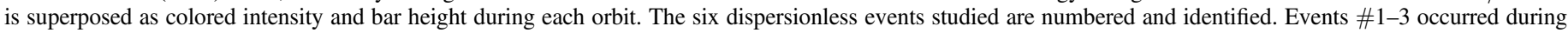

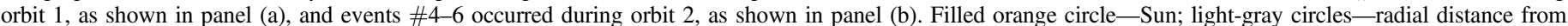
the Sun.

$+R$ direction; and (3) antisunward, defined here as all apertures with look directions $>120^{\circ}$ from the $+R$ direction. In general, the sunward look direction measures ions flowing away from the Sun, the antisunward look direction measures ions flowing toward the Sun, and the transverse look direction measures ions flowing perpendicular to the radial direction. Consequently, hereafter we refer to particles measured in the sunward look direction as those moving in the antisunward direction and those measured in the antisunward look direction as those flowing sunward. In Figures 3-8, the time histories of the differential intensities had at least one He triple coincidence count, while all the spectral slopes have relative uncertainty, assuming Poisson statistics, less than 50\%. In Figures 9 and 10 all data points have at least two triple coincidence counts.

\section{Observations}

\subsection{Overview of First Two Orbits}

During both orbits, IS $\odot$ IS measured a variety of particle intensity enhancements associated with local and remote acceleration sites (see McComas et al. 2019; Allen et al. 2020; Cohen et al. 2020; Giacalone et al. 2020; Hill et al. 2020; Joyce et al. 2020; Mitchell et al. 2020; Wiedenbeck et al. 2020). Many of these events, especially those measured by EPI-Lo inside $\sim 0.5 \mathrm{au}$, exhibit normal velocity dispersion during their onsets, as expected from propagation from the Sun to the spacecraft location following the simultaneous injection of ions with a broad range of energies or speeds from sources or acceleration regions near the Sun (e.g., Mazur et al. 1999; McComas et al. 2019; Hill et al. 2020; Leske et al. 2020). However, in this paper we identify and analyze six distinct $>0.03 \mathrm{MeV}$ nucleon ${ }^{-1} \mathrm{He}$ intensity enhancements that did not exhibit normal velocity dispersion during their onsets but instead showed that lower-energy or lower-speed ions arrived and peaked earlier than the higher-energy ions. For the sake of brevity, we hereafter refer to this effect as "inverse" velocity dispersion, although strictly speaking velocity dispersion is traditionally discussed only in terms of the onsets of particle events. These six events are most likely associated with stream interaction regions that are formed when fast solar wind streams overtake slower solar wind streams that were ejected earlier (see Allen et al. 2020; Cohen et al. 2020).

Figure 1 shows the two PSP orbits along with the locations where these six events were observed. Figure 1 also superposes the hourly averaged count rate of $>0.03 \mathrm{MeV}$ nucleon $^{-1} \mathrm{H}-\mathrm{Fe}$ ions observed by EPI-Lo during both orbits, with the color intensity and bar height representing the number of the counts. This count rate includes all $\mathrm{He}-\mathrm{Fe}$ triple coincident ion events identified by EPI-Lo, as well as all TOF-only ion events with energies less than $0.75 \mathrm{MeV}$ to exclude backgrounds due to galactic cosmic rays and other sources (see Hill et al. 2020).

Figure 2 identifies the six events in an overview plot that shows the EPI-Lo observations over the first two orbits: hourly averaged count rate from Figures 1 (panel (a)), 1/ion speed spectrogram versus time (panel (b)), and radial distance of PSP versus the longitude of the magnetic footpoint assuming a Parker spiral corresponding to $400 \mathrm{~km} \mathrm{~s}^{-1}$ for both orbits (panel (c)). In Figure 2(c), the color intensity superposed on the footpoint longitude represents the hourly averaged count rate from Figure 2(a). Table 1 lists Event \#/Orbit \# (Column (1)); year, start, and stop sampling times at PSP (Columns (2)-(4)); $P S P$ location (Column (5)); spectral fit parameters, the reduced chi-squared, and its statistical significance (Columns (6)-(10); see Section 3.3 for more details); the type of velocity dispersion, if any, observed during the onset (Column (11)); and the flow direction of $\sim 0.032-0.064 \mathrm{MeV}^{\text {nucleon }}{ }^{-1} \mathrm{He}$ ions in the spacecraft frame (Column (12)). Outside $\sim 0.25 \mathrm{au}$, $P S P$ undertakes significant data downlink and attitude control maneuvers, which reorients the spacecraft relative to the PSPSun line and thus changes the fields of view of the EPI-Lo apertures. For example, during events \#2-6, EPI-Lo had five apertures pointing within $60^{\circ}$ of the $+R$ direction, i.e., in the 

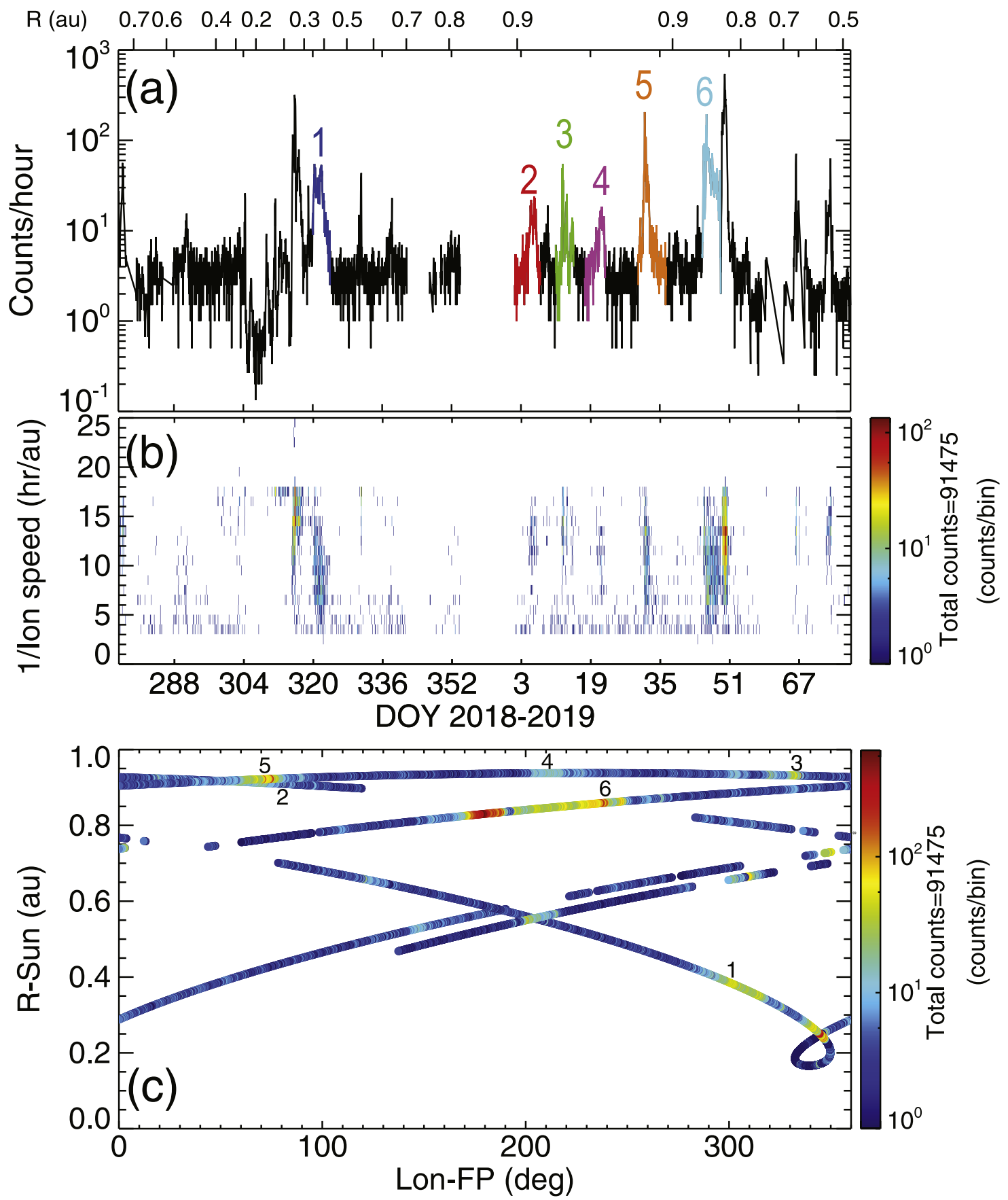

Figure 2. (a) Hourly averaged $\sim 0.03-2 \mathrm{MeV}$ nucleon ${ }^{-1} \mathrm{H}-\mathrm{Fe}$ count rate vs. time from EPI-Lo during the first two PSP orbits. (b) $1 /$ ion speed vs. time for $\sim 0.03-2 \mathrm{MeV}$ nucleon ${ }^{-1} \mathrm{H}-\mathrm{Fe}$ ions measured by EPI-Lo. (c) Radial distance of PSP from the Sun vs. longitude of the spacecraft's magnetic footpoint (assuming a Parker spiral corresponding to $400 \mathrm{~km} \mathrm{~s}^{-1}$ ). The $\sim 0.03-2 \mathrm{MeV}$ nucleon ${ }^{-1} \mathrm{H}-\mathrm{Fe}$ counts (color bar) are superposed, and count rate increases associated with the six events studied in this paper are numbered; time increases with decreasing footpoint longitude in each orbit.

sunward direction, 36 apertures pointing in the transverse direction, and 39 apertures in the antisunward direction. In contrast, during event \#1, as PSP moved from $\sim 0.31$ to $0.43 \mathrm{au}$, EPI-Lo had 17 apertures pointing in the sunward direction, 24 apertures pointing in the transverse look direction, and 39 apertures in the antisunward look direction. Thus, during all six events studied here, EPI-Lo is able to provide suprathermal $\mathrm{He}$ measurements in the three look directions defined above, and therefore potentially into their arrival directions from source region locations relative to the Sun and PSP.

\subsection{Time Histories of Intensities, Anisotropies, and Spectral Slopes}

\subsubsection{Event \#1 from Orbit 1: 2018, DOY 318-DOY 325}

EPI-Lo observed an He intensity enhancement between 2018, DOY 318-DOY 325 when PSP was at $\sim 0.35$ au. By this time $P S P$ had already completed its first perihelion pass. Figure 3(a) shows $4 \mathrm{hr}$ averages of the $\sim 0.03-1.99 \mathrm{MeV}$ nucleon $^{-1} \mathrm{He}$ intensities averaged over all 80 EPI-Lo apertures; Figure 3(b): $4 \mathrm{hr}$ averages of $\sim 0.031-0.062 \mathrm{MeV}$ nucleon $^{-1} \mathrm{He}$ intensities averaged over all apertures that measure particles 
Table 1

Sampling Times, Spectral Fit Parameters, and Flow Directions for Six $>0.03 \mathrm{MeV}$ nucleon ${ }^{-1}$ He Intensity Enhancements Associated with Stream Interaction Region Events Studied in This Paper

\begin{tabular}{|c|c|c|c|c|c|c|c|c|c|c|c|}
\hline \multirow[t]{2}{*}{$\begin{array}{l}\text { Event } \\
\text { \#/Orbit \# }\end{array}$} & \multirow[t]{2}{*}{ Year } & \multirow[t]{2}{*}{$\begin{array}{l}\text { Start } \\
\text { Time } \\
\text { (UT) }\end{array}$} & \multirow[t]{2}{*}{$\begin{array}{l}\text { Stop } \\
\text { Time } \\
\text { (UT) }\end{array}$} & \multirow[t]{2}{*}{ Location (au) } & \multicolumn{5}{|c|}{ Spectral Fit Parameters } & \multirow[t]{2}{*}{$\begin{array}{l}\text { Type of Velo- } \\
\text { city Dispersion }\end{array}$} & \multirow[t]{2}{*}{$\begin{array}{c}\text { Flow Direction of } \sim 0.032-0.064 \mathrm{MeV} \\
\text { Nucleon }{ }^{-1} \text { He Ions in Spacecraft } \\
\text { Frame }^{\text {c }}\end{array}$} \\
\hline & & & & & $j_{0}$ & $\gamma^{\mathrm{b}}$ & $E_{0}$ & $\chi_{v}^{2}$ & $P$ & & \\
\hline $1 / 1^{\mathrm{a}}$ & 2018 & $\begin{array}{c}\text { Nov } \\
15,2200\end{array}$ & $\begin{array}{c}\text { Nov } \\
20,0000\end{array}$ & 0.35 & $0.101 \pm 0.022$ & $0.914 \pm 0.084$ & $0.376 \pm 0.026$ & 0.965 & 0.517 & Inverse & $\mathrm{S} / \mathrm{T}$ \\
\hline $2 / 1$ & 2019 & $\begin{array}{c}\text { Jan } \\
1,0000\end{array}$ & $\begin{array}{c}\text { Jan } \\
7,1200\end{array}$ & 0.91 & $8.58 \times 10^{-5} \pm 6.29 \times 10^{-5}$ & $2.672 \pm 0.250$ & $\cdots$ & 0.689 & 0.498 & None & $\mathrm{S} / \mathrm{T}$ \\
\hline $3 / 1$ & 2019 & $\begin{array}{c}\text { Jan } \\
11,0000\end{array}$ & $\begin{array}{c}\text { Jan } \\
15,0400\end{array}$ & 0.932 & $3.3 \times 10^{-4} \pm 2.1 \times 10^{-4}$ & $2.185 \pm 0.223$ & $\cdots$ & 0.785 & 0.535 & None & $\mathrm{S} / \mathrm{T}$ \\
\hline $4 / 2$ & 2019 & $\begin{array}{c}\text { Jan } \\
17,1600\end{array}$ & $\begin{array}{c}\text { Jan } \\
22,1200\end{array}$ & 0.938 & $4.8 \times 10^{-4} \pm 3.6 \times 10^{-4}$ & $1.882 \pm 0.268$ & $\cdots$ & 0.713 & 0.510 & None & $\mathrm{S} / \mathrm{T}$ \\
\hline $5 / 2^{\mathrm{a}}$ & 2019 & $\begin{array}{c}\text { Jan } \\
30,0000\end{array}$ & $\begin{array}{c}\text { Feb } \\
5,1200\end{array}$ & 0.92 & $0.054 \pm 0.024$ & $0.748 \pm 0.224$ & $0.435 \pm 0.062$ & 0.958 & 0.505 & Inverse & $\mathrm{S}$ \\
\hline $6 / 2^{\mathrm{a}}$ & 2019 & $\begin{array}{c}\text { Feb } \\
13,1800\end{array}$ & $\begin{array}{c}\text { Feb } \\
18,0200\end{array}$ & 0.86 & $0.395 \pm 0.165$ & $0.437 \pm 0.197$ & $0.302 \pm 0.030$ & 0.956 & 0.503 & Inverse & $S$ \\
\hline
\end{tabular}

Notes.

${ }^{\mathrm{a}}$ Event also observed by EPI-Hi (see Cohen et al. 2020).

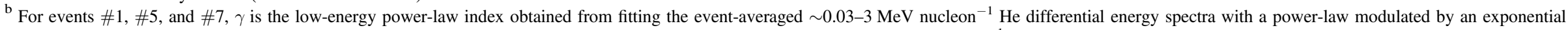
rollover (see Figure 10(a)), while for events \#2, \#3, and \#4 $\gamma$ is the power-law index obtained by fitting the $0.03-0.2 \mathrm{MeV}^{\text {nucleon }}{ }^{-1} \mathrm{He}_{\mathrm{H}}$ differential energy spectra (see Figure 10 (b))

${ }^{\mathrm{c}} \mathrm{S}=$ sunward flow; $\mathrm{T}=$ transverse flow. 


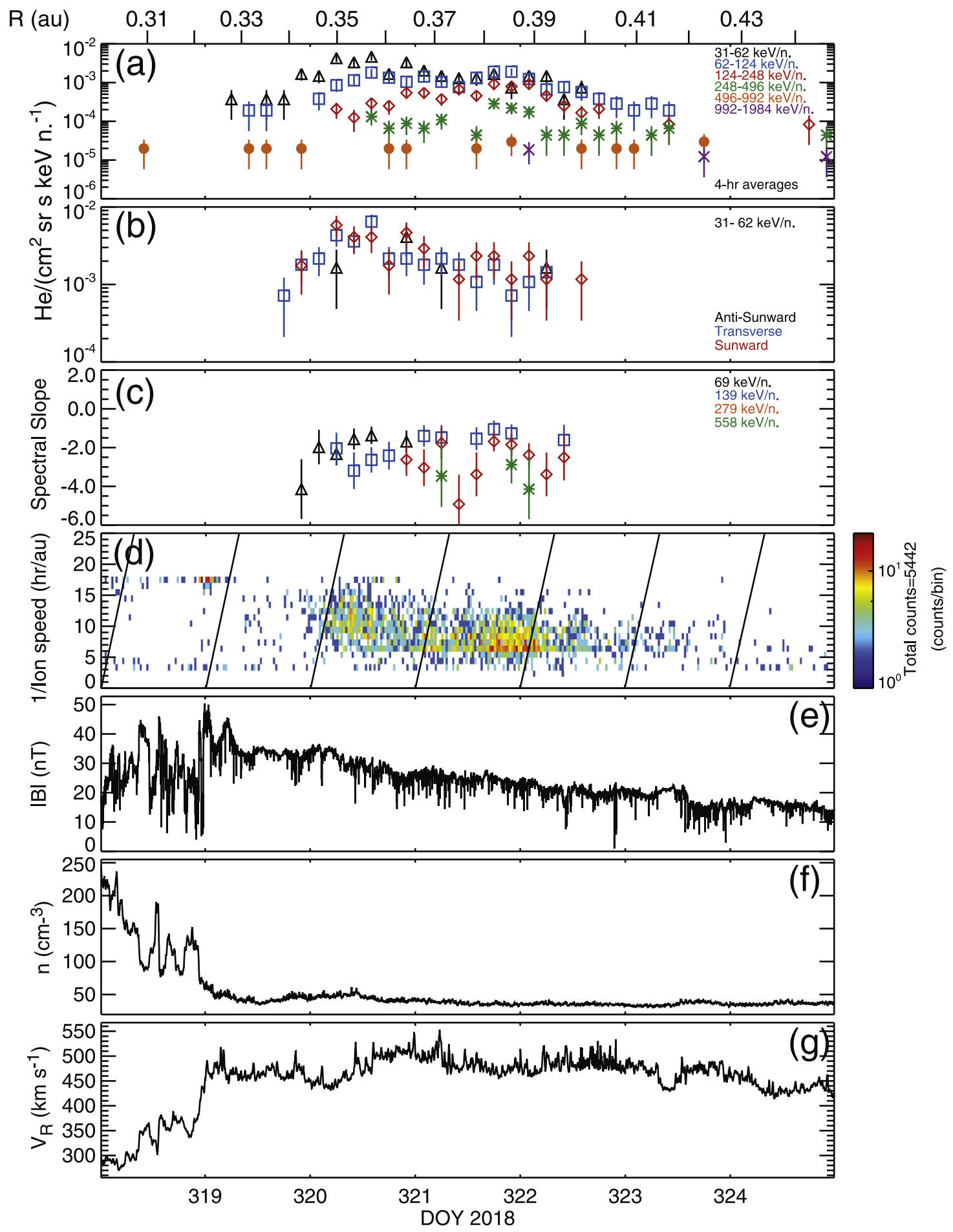

Figure 3. (a) $4 \mathrm{hr}$ averages of the $\sim 0.031-1.984 \mathrm{MeV}$ nucleon $^{-1} \mathrm{He}$ intensities averaged over all 80 apertures measured by IS $\odot$ IS/EPI-Lo on PSP during event \#1 from 2018, DOY 318-DOY 325. (b) $4 \mathrm{hr}$ averages of the $\sim 0.031-0.062 \mathrm{MeV}$ nucleon ${ }^{-1} \mathrm{He}$ intensities measured by EPI-Lo in three different flow directions (see text for more details); black = antisunward; blue = transverse; red = sunward. All EPI-Lo data shown here have at least one triple coincidence count. (c) Spectral slopes calculated using $4 \mathrm{hr}$ averages of the He intensities measured in pairs of adjacent energy bands shown in panel (a). Only slopes with relative uncertainty less than $50 \%$ are shown. (d) 1/ion speed vs. time for H-Fe counts measured by EPI-Lo. Ions that travel radially from the Sun to the location of PSP fall along slanted lines. (e, f, g) 1-minute averages of the magnetic field magnitude from PSP/FIELDS, solar wind density, and solar wind speed from PSP/SWEAP, respectively.

flowing in the antisunward, transverse, and sunward directions, as defined in Sections 2 and 3.1; Figure 3(c): the spectral slopes, $\gamma$, given by $\gamma=\log \left(j_{1} / j_{2}\right) / \log \left(E_{1} / E_{2}\right)$, where $j_{1}$ and $E_{1}$ are the average intensity and center energy (arithmetic mean) of the lower energy range, and $j_{2}$ and $E_{2}$ are the average intensity and center energy, respectively, of the next higher energy range shown in Figure 3(a); Figure 3(d): the 1/ion speed spectrogram versus time; Figures 3(e)-(g): 1-minute averages of the magnetic field magnitude from FIELDS and the 1-minute averages of the solar wind density and speed from SWEAP. 
Figures 3(e)-(g) show that the magnetic field magnitude and solar wind density become relatively steady as PSP exits an interaction region and encounters a moderately high speed $\left(\sim 500 \mathrm{~km} \mathrm{~s}^{-1}\right)$ solar wind stream on 2018, DOY 319 (Allen et al. 2020). The steady decline in the magnetic field magnitude and the relatively stable, low density through 2018, DOY 324 seen in Figures 3(e) and (f), respectively, indicate that PSP remained inside the rarefaction region throughout this period. Figure 3(a) clearly shows the following: (1) the $>0.03-$ $0.5 \mathrm{MeV}$ nucleon $^{-1} \mathrm{He}$ intensities increase by more than an order of magnitude when PSP was fully immersed in the highspeed solar wind stream from $\sim 1200$ UT on DOY 319; (2) starting from DOY 320, the He intensity peaks occur first at the lowest energies and then appear at successively higher energies at later times, with the $\sim 0.25-0.5 \mathrm{MeV}$ nucleon $^{-1} \mathrm{He}$ intensities peaking at $\sim 1200$ UT on DOY 321; and (3) from $\sim 1200$ UT on DOY 321, the $>0.062-0.25 \mathrm{MeV}$ nucleon $^{-1} \mathrm{He}$ intensities are either comparable to or greater than those seen at lower energies between $\sim 0.031$ and $0.062 \mathrm{MeV}_{\text {nucleon }}{ }^{-1}$. Figure 3(b) shows that the intensities of $\sim 0.031-0.062 \mathrm{MeV}$ nucleon $^{-1} \mathrm{He}$ ions flowing toward the Sun during the peak of the enhancement are either slightly greater than or comparable to those flowing in the antisunward and transverse directions. Figure 3(c) shows inverse velocity dispersion effects in that the spectral slope, $\gamma$, at $\sim 0.069 \mathrm{MeV}$ nucleon $^{-1}$ during the peak intensity enhancement on DOY 320 is approximately -2 , but it becomes close to -1 later in the event on DOY 321. In contrast, the $\gamma$ at higher energies remains $\approx-2$ throughout most of the event. Finally, Figure 3(d) shows the late arrival of higher-energy ions as a substantial increase in the counts per bin for ions with the highest speeds between DOY 321 and DOY 322, thereby exhibiting inverse velocity dispersion.

\subsubsection{Events \#2 and \#3 from Orbit 1 and Event \#4 from Orbit 2: 2019, DOY 1-DOY 23}

Figures 4(a)-(c) show $4 \mathrm{hr}$ averages of the He time intensity profiles and 1/ion speed spectrograms as in Figures 3(a)-(b) and (d), respectively, for the event seen on 2019, DOY 3-7, when PSP was at $\sim 0.92$ au. Figures 5 (a)-(c) show the same data during 2019, DOY 12-15 (event \#3). Figures 6(a)-(b) show the same data as in Figures 5(a) and (c), respectively, during 2019, DOY 17-23 (event \#4). The time intensity profiles in Figures 4(a) and 5(a) are different compared with those seen during event $\# 1$, in that the $\sim 0.031-0.062 \mathrm{MeV}$ nucleon $^{-1} \mathrm{He}$ intensity remains greater than that measured at

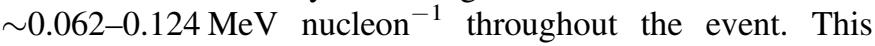
indicates that the corresponding spectrum does not turn over at the lowest energies. Figures 4(b) and 5(b) indicate an anisotropy in the transverse and sunward flow directions, with no counts measured along the antisunward flow direction. The spectral slopes are not shown here because the relative uncertainties are greater than 50\%. Figures 4(c), 5(c), and 6(b) show that all three events exhibited no velocity dispersion during the onsets. Finally, Like event $\# 1$, we note from Figure 6 (a) that the $\sim 0.031-0.062 \mathrm{MeV}$ nucleon ${ }^{-1} \mathrm{He}$ intensity is comparable to that measured between $\sim 0.062$ and $0.124 \mathrm{MeV}$ nucleon $^{-1}$ during the latter portion of the event, i.e., between 2019, DOY 21-DOY 22, i.e., the energy spectrum essentially flattens later in the event.

\subsubsection{Events \#5 and \#6 from Orbit 2: 2019, DOY 30-35, DOY 45-48}

Figure 7 shows $2 \mathrm{hr}$ averages of (a) the $\sim 0.031-0.992 \mathrm{MeV}$ nucleon $^{-1}$ He intensities, (b) the $\sim 0.031-0.062 \mathrm{MeV}_{\text {nucleon }}{ }^{-1}$ He anisotropy measurements, (c) the spectral slopes at $\sim 0.069$ and $0.139 \mathrm{MeV}$ nucleon $^{-1}$, and (d) the $1 /$ ion speed spectrograms during 2019, DOY 302-DOY 35 (event \#5). This event has similar properties to events $\# 2$ and $\# 3$, in terms of the relative behavior of the $\mathrm{He}$ intensities in the two lowest energy ranges, i.e., the $\sim 0.031-0.062 \mathrm{MeV}$ nucleon $^{-1} \mathrm{He}$ intensity remains greater than that measured at $\sim 0.062-0.124 \mathrm{MeV}$ nucleon $^{-1}$ throughout the event. Figure 7(b) shows that the $\mathrm{He}$ intensities are larger along the sunward flow direction compared to the transverse direction, with no counts detected in the antisunward flow direction. Figure 7(c) shows that the spectral slopes at the two lowest energies are comparable to $\gamma \approx-2$, within the uncertainties. Figure 7(d) shows hints of inverse velocity dispersion during the onset, as was also observed during event \#1 in Figure 3(d).

Figure 8 shows $2 \mathrm{hr}$ averages of (a) the $\sim 0.031-0.992 \mathrm{MeV}$ nucleon $^{-1} \mathrm{He}$ intensities, (b) the $\sim 0.031-0.062 \mathrm{MeV}^{\text {nucleon }}{ }^{-1}$ He anisotropy measurements, (c) the spectral slopes at $\sim 0.139$ and $\sim 0.279 \mathrm{MeV}^{\text {nucleon }}{ }^{-1}$, and (d) the $1 /$ ion speed spectrograms during 2019, DOY 44-DOY 49 (event \#6). Event \#6 has similar properties to those seen during event \#1, in terms of exhibiting inverse velocity dispersion during the onset and the relative behavior of the He intensities in the two lowest energy ranges, i.e., the $\sim 0.031-0.062 \mathrm{MeV}$ nucleon ${ }^{-1} \mathrm{He}$ intensity is comparable to that measured between $\sim 0.062$ and $0.124 \mathrm{MeV}$ nucleon $^{-1}$ throughout the event, indicating that the spectrum flattens at the lowest energies. Figure 8(b) shows that higher intensities are observed along the sunward flow direction compared to the transverse direction during the event. With the exception of the peak intensity periods of the event, no measurable counts are detected in the antisunward flow direction throughout most of the event. Figure 8(c) shows that during the peak of the event at $\sim 1800$ UT on DOY 45,2019 , the spectral slope at $\sim 0.139 \mathrm{MeV}$ nucleon $^{-1}$ is $\approx-1$ while that at $\sim 0.279 \mathrm{MeV}$ nucleon $^{-1}$ is between -2 and -4 , thus confirming that the energy spectrum flattens at lower energies. Note that the slopes at $\sim 0.069 \mathrm{MeV}$ nucleon $^{-1}$ are not plotted because they remained close to 0 throughout the event (also see Figure 10(a) and Table 1) and had relative uncertainties greater than $50 \%$.

\subsection{Event-averaged Differential Energy Spectra}

In order to further characterize the properties of each event, we obtain the event-averaged He differential energy spectra measured in the sunward, transverse, and antisunward flow directions and plot them in Figure 9. Since averaging over the entire event provides adequate counting statistics, we sum the triple coincidence He counts over energy ranges that differ by $\sqrt{ } 2$, e.g., $0.031-0.043 \mathrm{MeV}$ nucleon $^{-1}$, and so on. We only plot data points with at least two triple coincidence counts. Analyses of these energy spectra essentially confirm the observations discussed in Section 3.2 and can be summarized as follows: (1) For events \#1, \#5, and \#6, EPI-Lo measures higher intensities in the sunward and transverse flow directions compared with the antisunward flow direction, especially at the lowest energies. In all three flow directions during events \#1 and \#6, the spectra flatten or turn over at lower energies below 


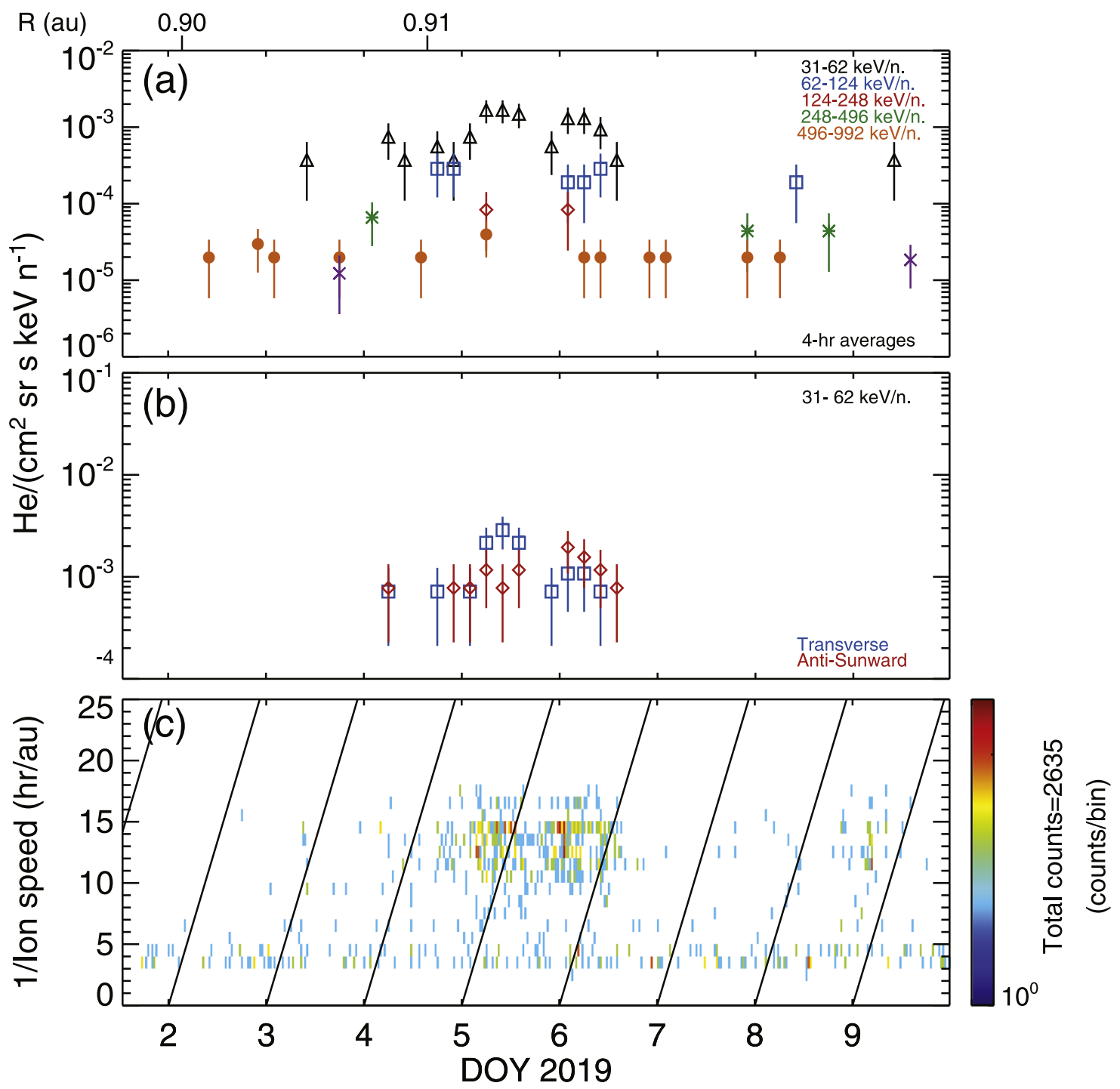

Figure 4. (a)-(c) Same as Figures 3(a), (b), and (d), using 4 hr averaged He data measured during event \#2 from 2019, DOY 1-DOY 10. The magnetic field and solar wind plasma data are not available during this time interval.

$\sim 0.2 \mathrm{MeV}$ nucleon $^{-1}$. In contrast, during event \#5, the spectra continue as single power laws down to $\sim 0.03 \mathrm{MeV}$ nucleon $^{-1}$. (2) In events \#2, \#3, and \#4, the He intensities in the sunward and transverse flow directions are comparable, while no measurable counts are detected in the antisunward direction - this is probably due to a combination of these events being weak and the fact that only five apertures are oriented in the sunward look direction. (3) In all events the energy spectra above $\sim 0.2 \mathrm{MeV}$ nucleon ${ }^{-1}$ appear to behave as power laws of the form $j \propto E^{-\gamma}$, where $\gamma \sim 2$.

Figure 10 compares the omnidirectional, event-averaged spectra in all six events. Also included in Figure 10(a) are data from EPI-Hi/LET (see Cohen et al. 2020), which observed events $\# 1, \# 5$, and \#6. Figure 10(b) shows the spectra for events \#2, \#3, and \#4 from EPI-Lo only, as these events are not observed by EPI-Hi. Solid curves in Figure 10(a) are fits to the spectra of the form $j=j_{0} E^{-\gamma} \exp \left(-E / E_{0}\right)$, where $j$ is the differential intensity at energy $E$ in $\mathrm{MeV}$ nucleon $^{-1}, j_{0}$ is the normalization constant, $\gamma$ is the power-law spectral index, and $E_{0}$ is the $e$-folding energy (Jones \& Ellison 1991). Solid lines in Figure $10(\mathrm{~b})$ are fits to the spectra of the form $j=j_{0} E^{-\gamma}$. All fits are obtained using the nonlinear least-squares LevenbergMarquardt technique and minimizing the chi-square $\chi^{2}$ value. The formal $1 \sigma$ uncertainty in each fit parameter is calculated using the off-diagonal terms of the covariance matrix (Markwardt 2009). The spectral fit parameters along with the reduced $\chi^{2}$ and its probability are listed in Table 1 .

We note that with the exception of event \#5, which has substantial uncertainties in the EPI-Lo data above $\sim 0.2 \mathrm{MeV}$ nucleon $^{-1}$, the fits for the remaining five events are reasonable, visually as well as statistically with $\sim 50 \%$ probabilities. It is noteworthy that the energy spectra during events \#1 and \#6 are remarkably similar even though $P S P$ was at heliocentric distances of $0.35 \mathrm{au}$ and $0.85 \mathrm{au}$, respectively.

\section{Discussion}

\subsection{Summary of Results}

We have studied the properties of suprathermal-throughenergetic $\sim 0.03-3 \mathrm{MeV}$ nucleon ${ }^{-1} \mathrm{He}$ ions associated with six corotating or stream interaction regions observed between $\sim 0.35$ and 0.95 au during the first two orbits of PSP. In 


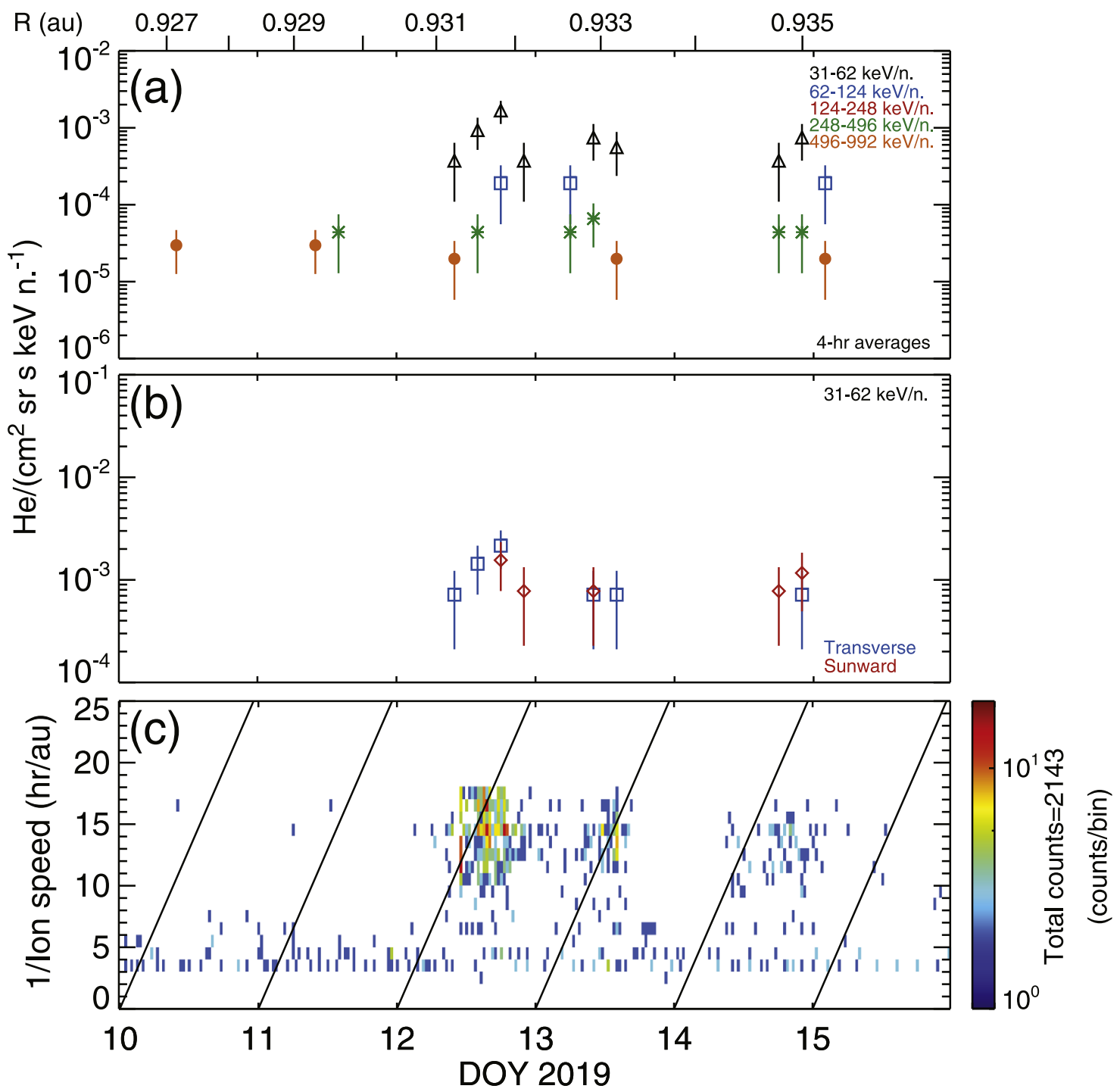

Figure 5. (a)-(c) Same as Figures 3(a), (b), and (d), using $4 \mathrm{hr}$ averaged He data measured during event \#3 from 2019, DOY 10-DOY 16. The magnetic field and solar wind plasma data are not available during this time interval.

particular, we examined time histories of the differential intensities, spectral slopes, and anisotropies in three different flow directions (sunward, transverse, and antisunward) between $\sim 0.03$ and $1 \mathrm{MeV}$ nucleon $^{-1}$, as well as the event-averaged differential energy spectra in these flow directions. We also fit the energy spectra for all events, including the three events observed above $\sim 1 \mathrm{MeV}$ nucleon $^{-1}$ by EPI-Hi (see Cohen et al. 2020). Our results are as follows:

1. In the two strongest SIR-associated enhancements seen at 0.35 and 0.85 au (events \#1 and \#6), the higher-energy ions arrive later during the events, and the event-averaged omnidirectional spectra are remarkably similar over a broad energy range from $\sim 0.031$ to $3 \mathrm{MeV}$ nucleon $^{-1}$. In event \#1, the He ions arrive when PSP was well away from the SIR trailing edge and entered the rarefaction region in the high-speed stream.

2. The He intensities in all SIR events either are nearly isotropic or show weak sunward anisotropies in the spacecraft frame.
3. In all events, the energy spectra between $\sim 0.2$ and $1 \mathrm{MeV}$ nucleon ${ }^{-1}$ behave as power laws of the form $\propto E^{-2}$. In the two strongest events, the energy spectra are well represented by power laws modulated by exponential rollovers. In these events, even though the spectra flatten at lower energies, they still continue as power laws down to $\sim 0.03 \mathrm{MeV}$ nucleon $^{-1}$. While the low-energy spectral index $\gamma$ exhibits significant event-to-event variability ranging from $\sim 0.43$ to 2.7 , the $e$-folding energies in events \#1, \#5, and \#6 have remarkably similar values between $\sim 0.3$ and $0.45 \mathrm{MeV}$ nucleon $^{-1}$.

\subsection{Sources of HE Ions Observed at PSP}

An important question is, what is the source of the suprathermal-through-energetic He ions seen in the PSP SIR events? Three possibilities are that (1) the $\mathrm{He}$ ions are accelerated locally, (2) the enhancements occur when PSP enters magnetic flux tubes that are already populated with ions accelerated at distant CIR shocks, and (3) the He ions originate 

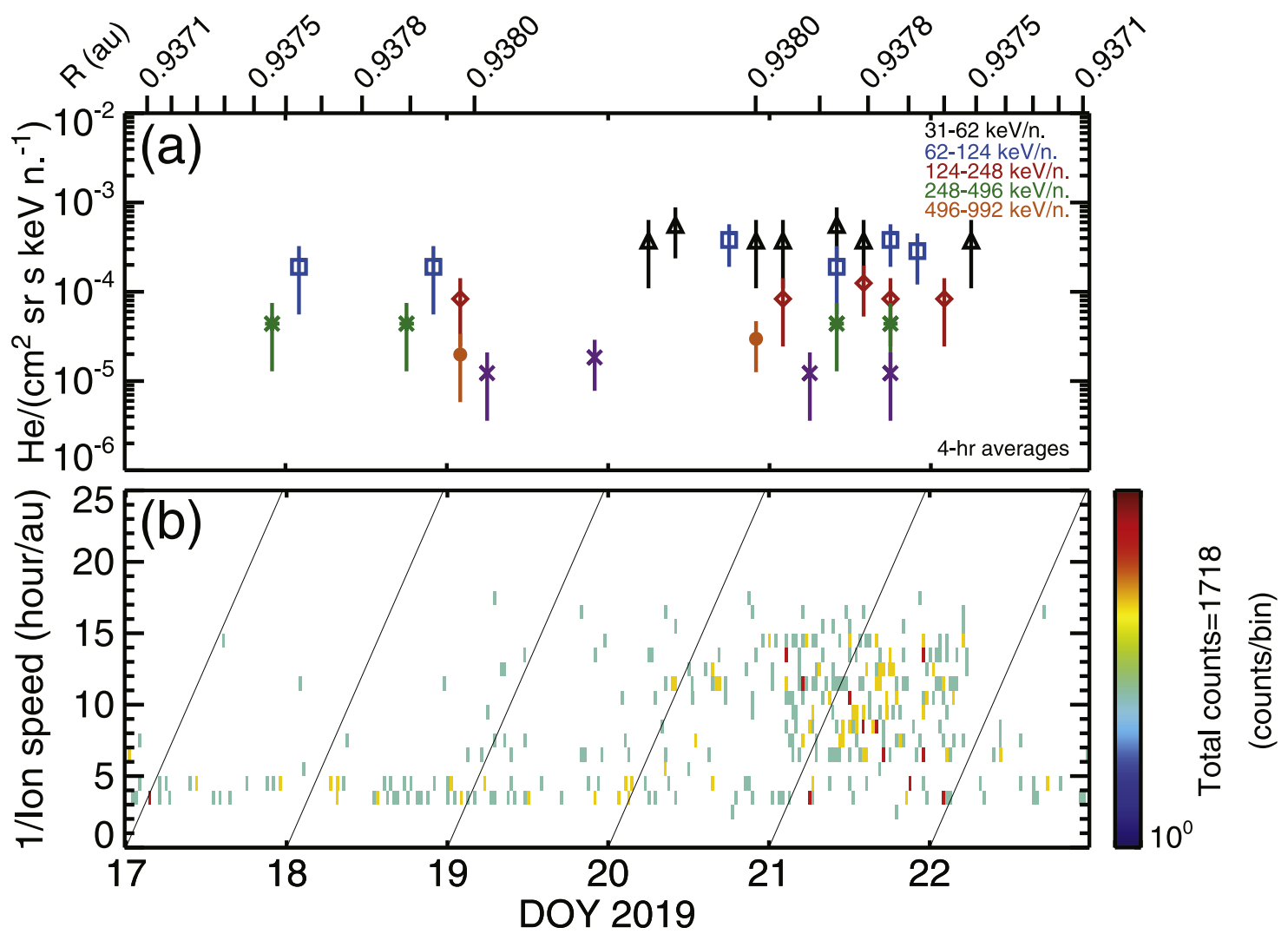

Figure 6. (a) and (b) Same as Figures 3(a) and (d), using $4 \mathrm{hr}$ averaged He data measured during event \#4 from 2019, DOY 17-DOY 23. The magnetic field and solar wind plasma data are not available during this time interval.

from evolving magnetic connection to distant CIR shocks. Since three of the events (\#2, \#3, and \#4) are relatively weak and the corresponding magnetic field and plasma data are not available, we restrict our discussion here to the three stronger events, \#1, \#5, and \#6.

Near-Earth and Ulysses observations at 5 au of CIR/SIRassociated particle events have shown that ions at suprathermal energies below $\sim 0.5 \mathrm{MeV}$ nucleon $^{-1}$ could be accelerated locally by second-order stochastic mechanisms (Schwadron et al. 1996), by compressional turbulence or waves (Fisk \& Gloeckler 2006; Jokipii \& Lee 2010; Zhang 2010), by magnetic islands (Zank et al. 2014; Khabarova et al. 2016; Zhao et al. 2018, 2019), or through mechanisms acting in strong compression regions (Giacalone et al. 2002; Chen et al. 2015). Three clear signatures of local particle acceleration are as follows: (1) the differential energy spectra continue to behave as power laws all the way down to energies as low as $\sim 0.03 \mathrm{MeV}$ nucleon $^{-1}$ (Mason et al. 1997, 2008, 2012), or the distribution functions merge smoothly into the solar wind ion distributions (Chotoo et al. 2000); (2) the peak He intensities occur at or near the trailing edges of the SIRs and are well correlated with the locally measured magnetic compression ratios regardless of whether the trailing edges are bounded by reverse shocks (Ebert et al. 2012a); and (3) the particle flow directions in the solar wind plasma frame reverse from antisunward to sunward near the trailing edge (Ebert et al. 2012b). Ebert et al. (2012b) also reported that sunward flows persisted throughout those events associated with weaker compression regions, thus indicating that suprathermal ions in many CIR/SIR-associated events observed at 1 au originate from sources located beyond Earth orbit.
We rule out local acceleration as a possible origin for the $\mathrm{He}$ ions in SIR-associated events on the basis of three key EPI-Lo observations presented here, namely, (1) the He intensities at all energies peak inside the rarefaction region and not near the trailing edge of the SIR that was observed at $\sim 0.35 \mathrm{au}$; (2) above $\sim 0.2 \mathrm{MeV}$ nucleon $^{-1}$, the He intensities in both events are nearly isotropic or exhibit weak sunward anisotropy with no flow reversals, which implies that the ions are essentially flowing inward toward the Sun; and (3) the energy spectra flatten below $\sim 0.2 \mathrm{MeV}$ nucleon ${ }^{-1}$ and the higher-energy ions arrive later during the events, which indicate that the ion populations originate from sources that strengthen with time. We remark that most of the nonshock or nontrailing edge associated local particle acceleration mechanisms typically require strong scattering or trapping and confinement, which should generate isotropic particle distributions in the plasma frame (e.g., Schwadron et al. 1996; Zank et al. 2014; Khabarova et al. 2016), and that this is somewhat at odds with our observations that show nearly isotropic distributions or sunward flows in the spacecraft frame.

Figure 2 shows that during events \#1 and \#6 PSP was magnetically connected to separate footpoint longitudes on the Sun, i.e., $300^{\circ}$ versus $230^{\circ}$, and so the corresponding SIRs are likely to have distinct solar source regions. Thus, it is unlikely that during these two events PSP entered the same rarefaction region or magnetic flux tube that is already populated with ions accelerated at the same distant CIR shock. However, the fact that both events exhibit nearly isotropic distributions in the spacecraft frame could also be interpreted in favor of the notion that $P S P$ may have entered separate rarefaction regions that are already filled with CIR-shock-accelerated populations. We rule 


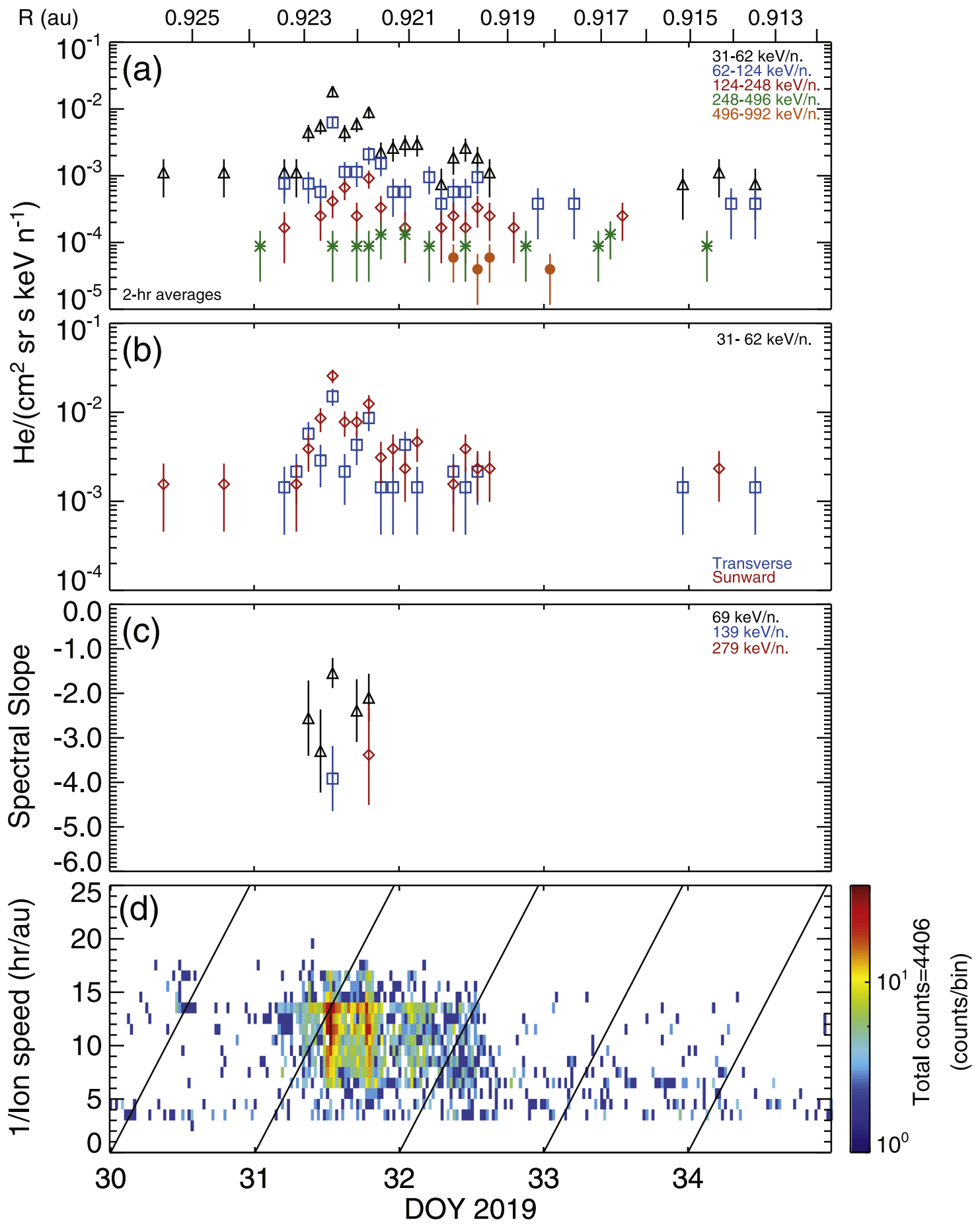

Figure 7. (a)-(d) Same as Figures 3(a)-(d), but using 2 hr averaged He data measured during event \#5 from 2019, DOY 30-DOY 35. The magnetic field and solar wind plasma data are not available during this time interval.

out this possibility based on the observation that the higherenergy ions arrive much later in the event, which instead points to an evolving magnetic connection either to a distant CIR shock that strengthens with time or to increasingly stronger, more efficient portions of the distant CIR shock with time. Finally, since event \#5 actually exhibits a net sunward anisotropy throughout the event with no flow reversals, we can rule out potential contributions from local acceleration processes, as well as the possibility that $P S P$ entered a rarefaction region filled with accelerated particles. Thus, for events $\# 1, \# 5$, and $\# 6$, we are left with the only possibility that the $\mathrm{He}$ ions originate from a CIR shock beyond the location of PSP.

Events \#1, \#5, and \#6 were also observed by EPI-Hi and interpreted by Cohen et al. (2020) as "classic" CIR/SIRassociated particle events in which the $>2 \mathrm{MeV}$ nucleon $^{-1}$ proton and $\mathrm{He}$ intensity enhancements are unlikely to be produced by local acceleration processes. Strong support for a distant source rather than local acceleration processes for the suprathermal $\mathrm{He}$ ions observed in event $\# 1$ is provided by two key results shown here: (1) the $<1 \mathrm{MeV}$ nucleon $^{-1} \mathrm{He}$ intensity enhancements are observed well after the compression 

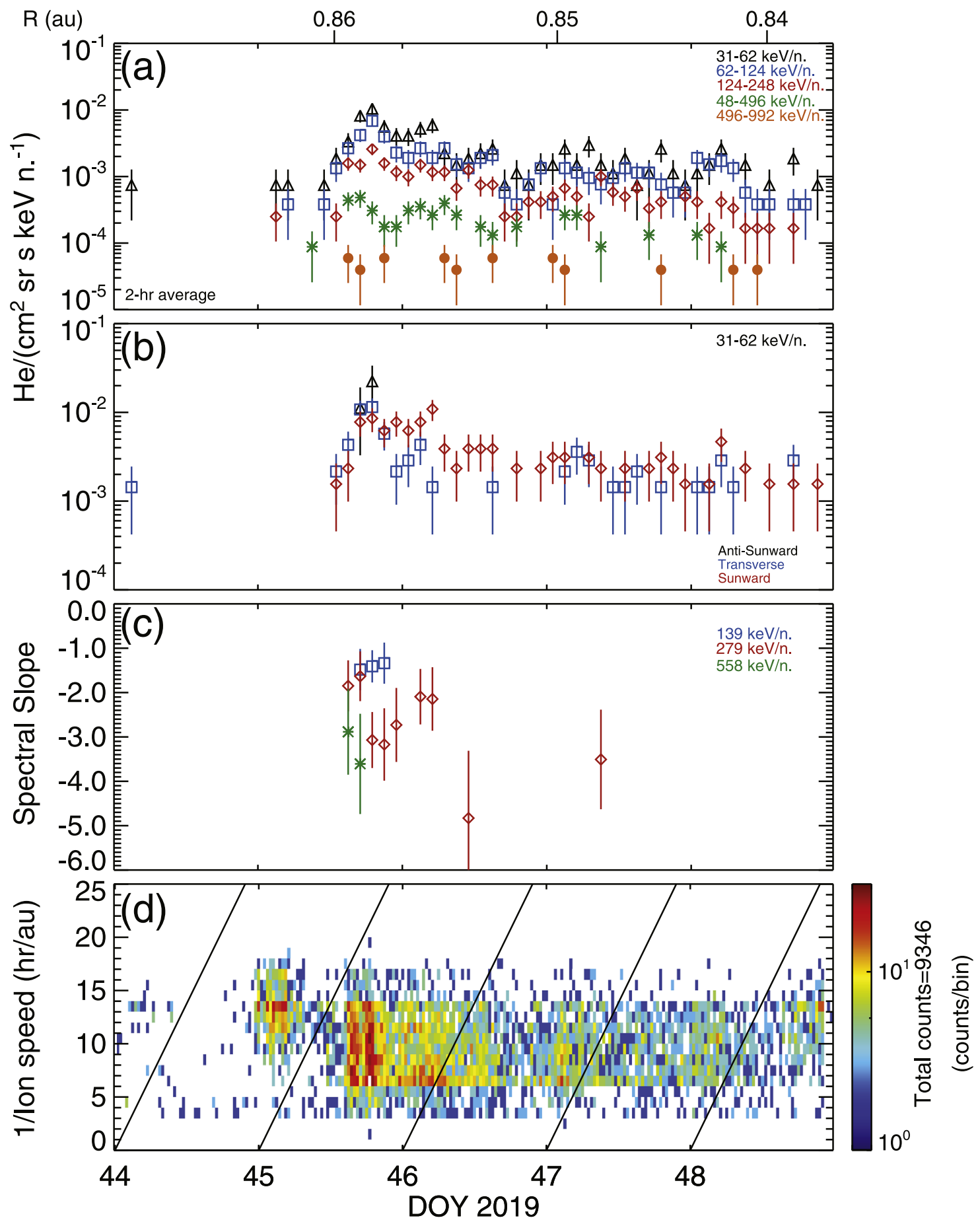

Figure 8. (a)-(d) Same as Figures 3(a)-(d), but for event \#6 from 2019, DOY 44-DOY 49.

region trailing edge had corotated past and when $P S P$ was immersed in the high-speed stream or inside the rarefaction region (e.g., Allen et al. 2020), and (2) the higher-energy ions arrive and maximize significantly later than the lower-energy ions. Similar energy-dependent temporal behavior was also observed by Reames et al. (1997) during a CIR event at 1 au and was interpreted in terms of the Wind spacecraft getting magnetically connected to stronger, more efficient acceleration portions of the distant reverse shock later during the event.

It is worth noting that more recently Zhao et al. (2016) extended the Fisk \& Lee (1980) model and included particle acceleration and transport to simulate the He differential energy spectra in a CIR event that was observed at STEREO-A, $S T E R E O-B$, and $A C E$. They concluded that local acceleration occurs at STEREO-A, where a reverse shock bounded the trailing edge. Based on the relative shapes of the He spectra, which either flattened or turned over below $\sim 0.5 \mathrm{MeV}$ nucleon $^{-1}$, Zhao et al. (2016) estimated that the particles seen at $A C E$ and STEREO-B most likely originated from the same CIR shock that had subsequently moved out to $\sim 1.73$ and $\sim 3.63 \mathrm{au}$, respectively, from the Sun. Putting the PSP observations of SIR-associated ions at $\sim 0.35$ au in context of the results of Zhao et al. (2016), we point out two new and surprising results, namely, (1) the He intensity-time profiles 

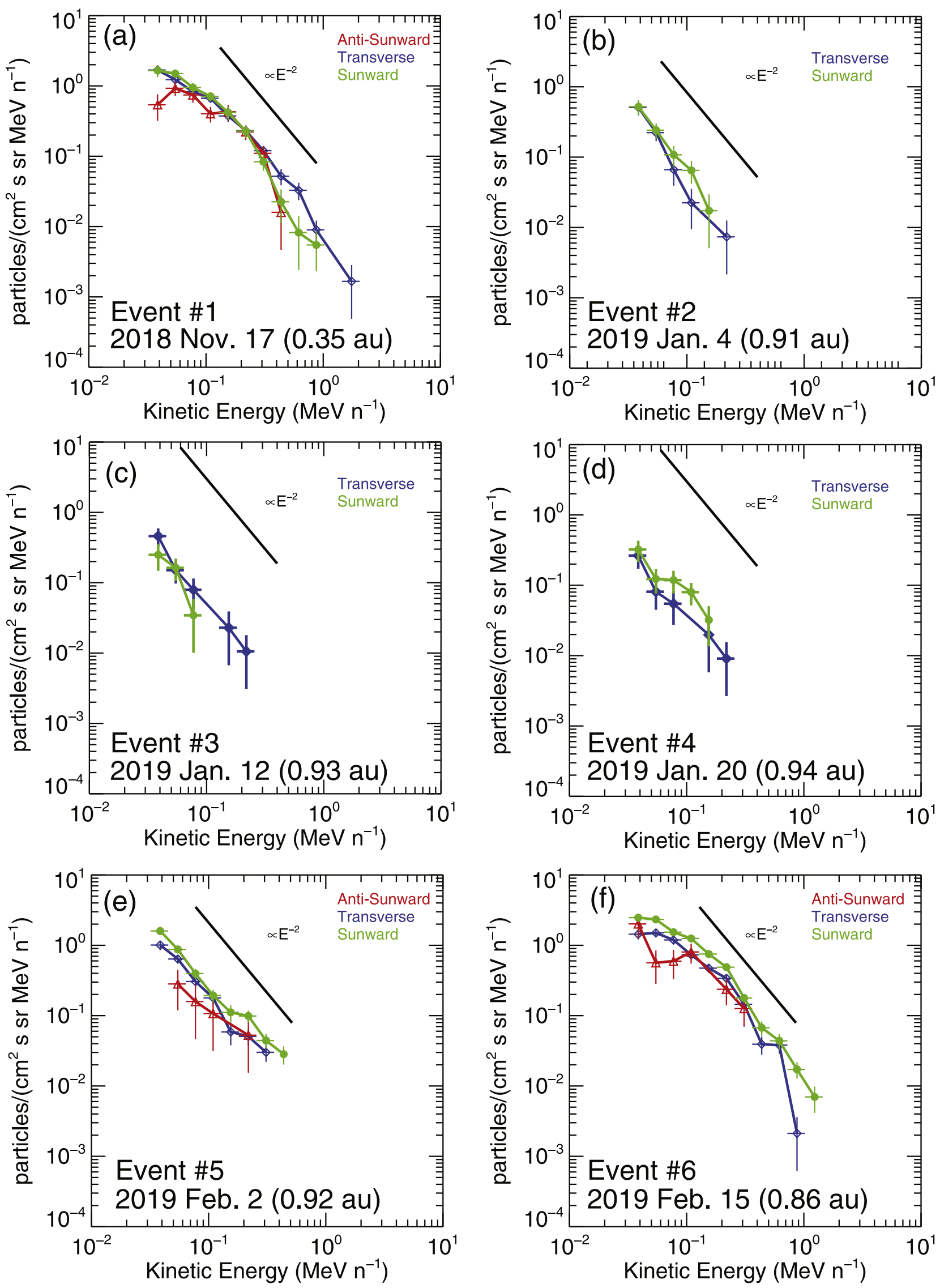

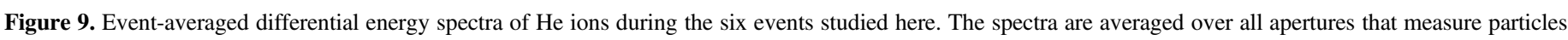

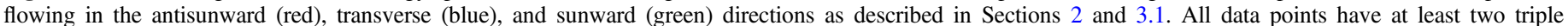

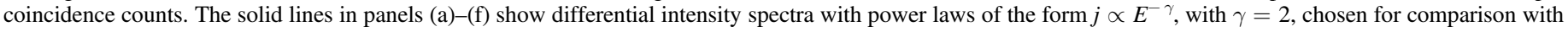
the event-averaged spectra. 

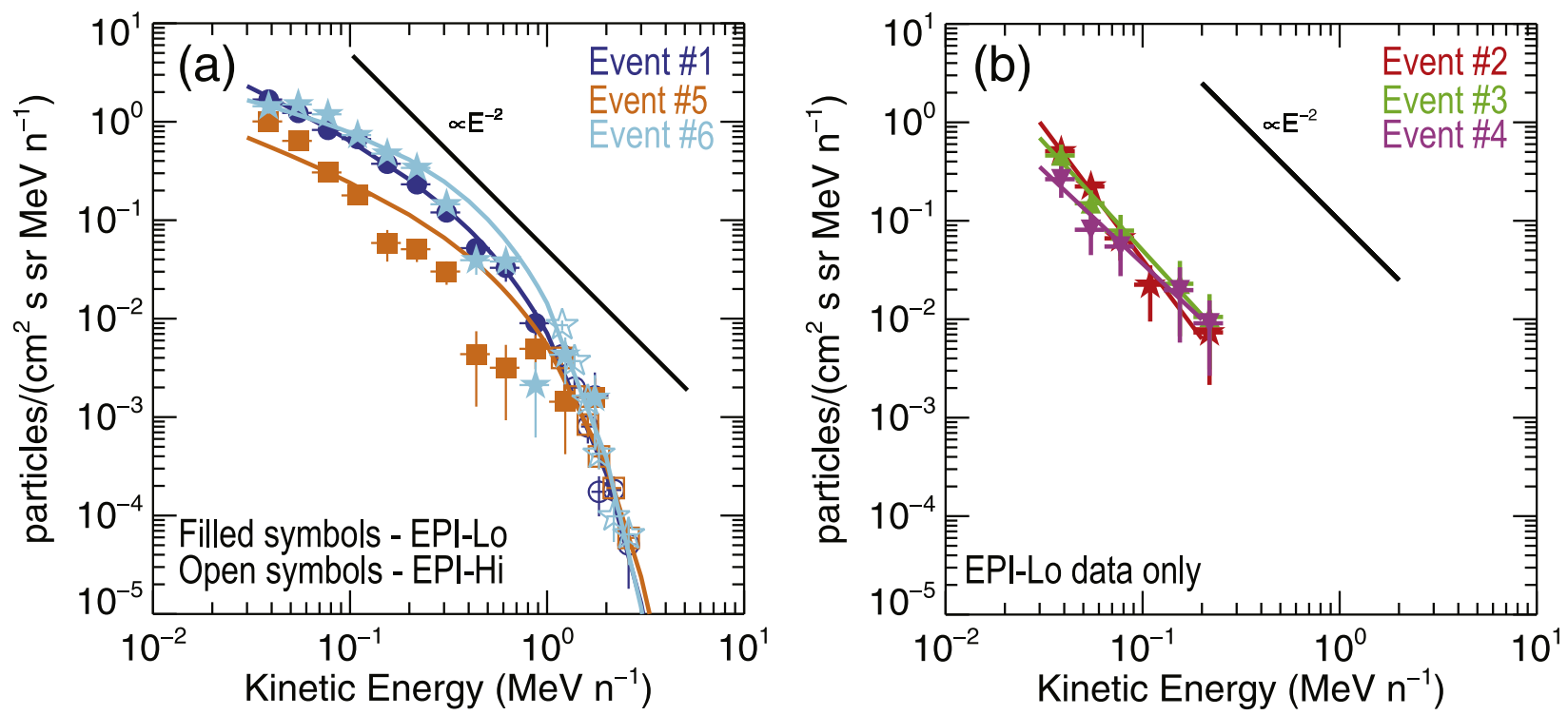

Figure 10. Omnidirectional, event-averaged He differential energy spectra during the six events studied here. (a) Events \#1, \#5, and \#6 are detected by both EPI-Lo and EPI-Hi. (b) Events \#2, \#3, and \#4 are detected by EPI-Lo only. Solid lines show (a) results of power laws modulated by exponential fits to the data and (b) power-law fits to the data. The fit parameters and their uncertainties and the reduced chi-squared and its statistical significance for each fit are provided in Table 1.

and the event-averaged energy spectra at 0.35 au are similar to those measured during a separate event, event \#6, seen at $0.85 \mathrm{au}$, and (2) the spectrum flattens but does not turn over as expected from energy losses due to adiabatic deceleration during transport from an SIR shock located well beyond 1 au. This indicates either that the SIR shock was located closer to PSP or that traditional transport models overestimate the energy losses due to adiabatic deceleration. Based on the result that the energy spectra did not flatten at the lowest energies in event \#5, we suggest that the shock in this case was probably not too far from PSP. Finally, we remark that particles accelerated at shocks located between $\sim 2$ and 3 au have to travel longer path lengths owing to the spiral winding of the interplanetary magnetic field (IMF) and could, as a result, undergo substantial adiabatic energy losses. In contrast, those particles accelerated at shocks located just beyond 1 au travel along an increasingly radial IMF configuration to reach the location of PSP at 0.35 au and thus may not suffer significant adiabatic cooling.

We also point out the possibility that rarefaction regions in high-speed streams could facilitate easier transport through the inner heliosphere and/or that particles that travel within such regions do not undergo significant energy losses due to adiabatic deceleration. Indeed, evidence that particle transport may involve less pitch-angle scattering and is therefore substantially easier within such rarefaction regions has been presented by, for example, McDonald \& Burlaga (1985), who referred to these regions as "magnetic channels" in which solar energetic particles or SEPs accelerated near the Sun and Jovian electrons can propagate somewhat more easily to Earth orbit (e.g., Mewaldt et al. 1976; Chenette 1980). Additional clues regarding the ease with which particles accelerated at remote CIR shocks can propagate over large distances without suffering significant adiabatic energy losses was provided by Roelof et al. (1996), who noted the clock-like regularity in the appearance of recurrent particle enhancements when Ulysses was immersed in the high-speed solar wind flows at higher latitudes even though no local compression regions were observed. One reason why particles may not suffer strong adiabatic energy losses and undergo significantly less pitchangle scattering in the rarefaction regions is that the high-speed solar wind comprises lower turbulence levels compared to those present in the low-speed wind (e.g., Bruno \& Carbone 2013). In a future study, we will model the energy spectra to estimate shock locations for events \#1, \#5, and \#6; improve estimates of energy loss from adiabatic deceleration using the "reservoir" concept (e.g., Roelof 2015); and simulate particle transport in magnetic channels inside rarefaction regions (e.g., Anderson et al. 1992; Armstrong et al. 1994; Maia et al. 1998). In particular, we will model the combined the EPI-Lo and EPI-Hi energy spectra for events \#1, \#5, and \#6 (Figure 10(a)), as well as the corresponding He energy spectra measured in the four events (events $\# 1, \# 2$, $\# 5$, and $\# 6$ ) that were also detected subsequently at STEREO-A.

\section{Conclusions}

The $\sim 0.03-3 \mathrm{MeV}$ nucleon ${ }^{-1} \mathrm{He}$ energy spectra associated with SIRs observed at $\sim 0.35$ and $\sim 0.85$ au are identical-they behave as flat power laws modulated by exponential rollovers with $e$-folding energies of $\sim 0.4 \mathrm{MeV}$ nucleon $^{-1}$ and exhibit near-isotropy and or sunward flows in the spacecraft frame throughout the event durations. Our new results provide evidence that suprathermal He ions observed in six CIR/SIR events by IS $\odot$ IS originate from sources or shocks beyond the location of PSP rather than from acceleration processes occurring at the locally observed compression regions (e.g., Giacalone et al. 2002). Our results also suggest that rarefaction regions that typically follow the SIRs may facilitate easier particle transport throughout the inner heliosphere, particularly between $\sim 0.35$ and 0.85 au in the ecliptic plane, where lowenergy ions do not undergo as significant energy losses due to adiabatic deceleration as has been predicted by existing models (Fisk \& Lee 1980; Zhao et al. 2016). Finally, we remark that although we have only observed one SIRassociated suprathermal-through-energetic He ion event inside $\sim 0.5$ au, the PSP observations reported here pose a serious challenge for current particle transport models that predict 
turnovers in the energy spectra at lower energies below $\sim 0.5 \mathrm{MeV}$ nucleon $^{-1}$ due to adiabatic energy losses.

This work was supported by NASA's Parker Solar Probe Mission, contract NNN06AA01C. We thank all the scientists and engineers who have worked hard to make PSP a successful mission, in particular the engineers, scientists, and administrators who designed and built IS $\odot$ IS $/$ EPI-Lo, IS $\odot$ IS $/$ EPI-Hi, FIELDS, and SWEAP instrument suites and support their operations and the scientific analysis of its data. For their contributions to the scientific configuration and instrumental analysis, we owe special thanks to P. Kollmann, J. Peachy, and J. Vandegriff at JHU/APL for EPI-Lo and B. Kecman and W. R. Cook at Caltech for EPI-Hi. The IS $\odot$ IS data are available at http://spp-isois.sr.unh.edu/data_public/, as well as at the NASA Space Physics Data Facility. Work at SwRI is also supported in part under NASA grants 80NSSC17 K0009, NNX15AG09G，80NSSC18K0520, NNX17AI17G, 80NSSC19K0079, and NNX13AI75G. S.D.B. acknowledges support of the Leverhulme Trust Visiting Professorship program.

\section{ORCID iDs}

M. I. Desai (iD https:// orcid.org/0000-0002-7318-6008

D. G. Mitchell (i) https://orcid.org/0000-0003-1960-2119

J. R. Szalay (i) https://orcid.org/0000-0003-2685-9801

M. E. Hill (ib https://orcid.org/0000-0002-5674-4936

D. J. McComas (i) https://orcid.org/0000-0001-6160-1158

E. R. Christian (1D https://orcid.org/0000-0003-2134-3937

N. A. Schwadron (i) https://orcid.org/0000-0002-3737-9283

C. Joyce (iD https://orcid.org/0000-0002-3841-5020

R. W. Ebert (10) https://orcid.org/0000-0002-2504-4320

M. A. Dayeh (D) https://orcid.org/0000-0001-9323-1200

R. C. Allen (i) https://orcid.org/0000-0003-2079-5683

R. A. Leske (iD https://orcid.org/0000-0002-0156-2414

W. H. Matthaeus (iD https://orcid.org/0000-0001-7224-6024

S. D. Bale (i) https://orcid.org/0000-0002-1989-3596

M. Pulupa (i) https://orcid.org/0000-0002-1573-7457

R. J. MacDowall (ID https://orcid.org/0000-0003-3112-4201

J. C. Kasper (iD https://orcid.org/0000-0002-7077-930X

\section{References}

Allen, R. C., Lario, D., Odstrčil, D., et al. 2020, ApJS, doi:10.3847/15384365/ab578f

Anderson, K. A., Chaizy, P., Lin, R. P., \& Sommers, J. 1992, GeoRL, 19, 1283 Armstrong, T. P., Haggert, D., Lanzerotti, L. J., et al. 1994, GeoRL, 21, 1747 Bale, S. D., Goetz, K., Harvey, P. R., et al. 2016, SSRv, 204, 49

Barnes, C. W., \& Simpson, J. A. 1976, ApJL, 210, L91

Bruno, R., \& Carbone, V. 2013, LRSP, 10, 2

Bryant, D. A., Cline, T. L., Desai, U. D., \& McDonald, F. B. 1963, PhRvL, 11,144

Chen, J. H., Schwadron, N. A., Möbius, E., \& Gorby, M. 2015, JGRA, 120, 9269

Chenette, D. L. 1980, JGR, 85, 2243

Chotoo, K., Schwadron, N. A., Mason, G. M., et al. 2000, JGR, 105, 23107
Cohen, C. M. S., Christian, E. R., Cummings, A. C., et al. 2020, ApJS, doi:10. 3847/1538-4365/ab4c38

Desai, M. I., Marsden, R. G., Sanderson, T. R., et al. 1997, GeoRL, 24, 1155 Desai, M. I., Marsden, R. G., Sanderson, T. R., et al. 1999, JGR, 104, 6705

Ebert, R. W., Desai, M. I., Dayeh, M. A., \& Mason, G. M. 2012a, ApJ, 749, 73 Ebert, R. W., Desai, M. I., Dayeh, M. A., \& Mason, G. M. 2012b, ApJL, 754, L30

Fisk, L. A., \& Gloeckler, G. 2006, ApJL, 640, L79

Fisk, L. A., \& Lee, M. A. 1980, ApJ, 237, 620

Fox, N. J., Velli, M. C., Bale, S. D., et al. 2016, SSRv, 204, 7

Giacalone, J., Jokipii, J. R., \& Kota, J. 2002, ApJ, 573, 845

Giacalone, J., Mitchell, D. G., Allen, R. C., et al. 2020, ApJS, doi:10.3847/ $1538-4365 / a b 5221$

Gloeckler, G., Hovestadt, D., \& Fisk, L. A. 1979, ApJL, 230, L191

Gosling, J. T., Bame, S. J., McComas, D. J., et al. 1993, GeoRL, 20, 2789

Gosling, J. T., Borrini, G., Asbridge, J. R., et al. 1981, JGR, 86, 5438

Hill, M. E., Mitchell, D. G., Allen, R. C., et al. 2020, ApJS, doi:10.3847/1538$4365 /$ ab643d

Hill, M. E., Mitchell, D. G., Andrews, G. B., et al. 2017, JGRA, 122, 1513

Jokipii, J. R., \& Lee, M. A. 2010, ApJ, 713, 475

Jones, F. C., \& Ellison, D. C. 1991, SSRv, 58, 259

Joyce, C. J., McComas, D. J., Christian, E. R., et al. 2020, ApJS, doi:10.3847/ $1538-4365 / \mathrm{ab} 5948$

Kasper, J. C., Abiad, R., Austin, G., et al. 2016, SSRv, 204, 131

Khabarova, O., Zank, G. P., Li, G., et al. 2016, ApJ, 827, 122

Leske, R. A., Christian, E. R., Choen, C. M. S., et al. 2020, ApJS, doi:10.3847/ $1538-4365 /$ ab5712

Maia, D., Malandraki, O., Pick, M., et al. 1998, JGR, 103, 9545

Markwardt, C. B. 2009, in ASP Conf. Ser. 411, Astronomical Data Analysis Software and Systems XVIII, ed. D. Bohlender, P. Dowler, \& D. Durand (San Francisco, CA: ASP), 251

Mason, G. M., Desai, M. I., \& Li, G. 2012, ApJL, 748, L31

Mason, G. M., Leske, R. A., Desai, M. I., et al. 2008, ApJ, 678, 1458

Mason, G. M., Mazur, J. E., Dwyer, J. R., Reames, D. V., \& von Rosenvinge, T. T. 1997, ApJL, 486, L149

Mazur, J. E., Mason, G. M., Looper, M. D., Leske, R. A., \& Mewaldt, R. A. 1999, GeoRL, 26, 173

McComas, D. J., Alexander, N., Angold, N., et al. 2016, SSRv, 204, 187

McComas, D. J., Christian, E. R., Cohen, C. M. S., et al. 2019, Natur, 576, 223

McDonald, F. B., \& Burlaga, L. F. 1985, Proc. ICRC, 4, 346

McDonald, F. B., Teegarden, B. J., Trainor, J. H., von Rosenvinge, T. T., \& Webber, W. R. 1976, ApJL, 203, L149

Mewaldt, R. A., Stone, E. C., \& Vogt, R. E. 1976, JGR, 81, 2397

Mitchell, Giacalone, J., Allen, R. C., et al. 2020, ApJS, doi:10.3847/1538$4365 / \mathrm{ab} 63 \mathrm{cc}$

Reames, D. V., Ng, C. K., Mason, G. M., et al. 1997, GeoRL, 24, 2917

Richardson, I. G. 2018, LRSP, 15, 1

Richardson, I. G., Barbier, L. M., Reames, D. V., \& von Rosenvinge, T. T. 1993, JGR, 98, 13

Roelof, E. C. 2015, J. Phys. Conf. Ser., 642, 012023

Roelof, E. C., Simnett, G. M., \& Tappin, S. J. 1996, A\&A, 316, 481

Schwadron, N. A., Fisk, L. A., \& Gloeckler, G. 1996, GeoRL, 23, 2871

Simnett, G. M., Sayle, K., Roelof, E. C., \& Tappin, S. J. 1994, GeoRL, 21,1561

Smith, E. J., \& Wolfe, J. H. 1976, GeoRL, 3, 137

Van Hollebeke, M. A. I., McDonald, F. B., Trainor, J. H., \& von Rosenvinge, T. T. 1978, JGR, 83, 4723

Vourlidas, A., Howard, R. A., Plunkett, S. P., et al. 2016, SSRv, 204, 83

Wiedenbeck, Bucik, R., Mason, G. M., et al. 2020, ApJS, doi:10.3847/15384365/ab5963

Zank, G. P., le Roux, J. A., Webb, G. M., Dosch, A., \& Khabarova, O. 2014, ApJ, 797, 28

Zhang, M. 2010, JGR, 115, A12102

Zhao, L., Li, G., Ebert, R. W., et al. 2016, JGRA, 121, 77

Zhao, L.-L., Zank, G. P., Chen, Y., et al. 2019, ApJ, 872, 4

Zhao, L.-L., Zank, G. P., Khabarova, O., et al. 2018, ApJL, 864, 34 